\title{
EL PROCESO DE APRENDIZAJE DE FRANCÉS CIENTÍFICO-TÉCNICO EN LA UNIVERSIDAD: ENSEÑAR AL ESTUDIANTE A PENSAR
}

\author{
Ignacio Iñarrea Las Heras \\ Universidad de La Rioja
}

\begin{abstract}
RESUMEN: En este artículo se presenta una propuesta de trabajo docente en el aula, realizada con la asignatura Francés Técnico, perteneciente al plan de estudios de la Licenciatura en Enología (Universidad de La Rioja). El objetivo de la misma es enseñar al estudiante a desarrollar una forma de pensamiento que le permita ser consciente de lo que realmente sabe e ignora sobre dicha materia. Asimismo, se pretende que el estudiante adquiera un dominio del idioma específico en el cual el pensamiento convergente actúe con rapidez y agilidad desde un estilo de pensamiento ejecutivo.
\end{abstract}

PALABRAS CLAVE: Francés científico-técnico, enseñar a pensar, metacognición, pensamiento convergente, estilo de pensamiento ejecutivo.

\section{THE PROCESS OF LEARNING FRENCH FOR SCIENCE AT UNIVERSITY: TEACHING STUDENTS THINK}

\begin{abstract}
This paper presents a proposal for the teaching of French for Science in the Degree of Enology (Universidad de La Rioja). The main objective of the proposal is to develop in the student an awareness of what he/she knows or does not know. Likewise, we intend the student to acquire a mastery of the language in which convergent thinking works swiftly and promptly from an executive thinking style.

KEYWORDS: French for Science, teaching to think, metacognition, convergent thinking, executive thinking style.

Recibido: 18/07/2011

Aceptado: 08/02/2012
\end{abstract}

\section{INTRODUCCIÓN}

En las actuales circunstancias de la universidad española, en que se están implantando los nuevos planes de estudios al objeto de adecuarse al marco del Espacio Euro- 
peo de Educación Superior, la concepción de la actividad docente y del aprendizaje han cambiado sustancialmente Ahora más que nunca, el estudiante es el protagonista de su propia formación. Por ello, y como es bien sabido, la adquisición de aptitud para trabajar de forma autónoma es un elemento clave en la consecución de las competencias establecidas en las diferentes titulaciones de Grado. Para el desarrollo de esta autonomía es esencial conseguir activar y potenciar en el alumnado la metacognición ${ }^{1}$ Ésta se podría definir, básicamente, como la capacidad necesaria para conocer las propias destrezas y carencias, los conocimientos y lagunas en las distintas disciplinas que se aborden, así como para determinar cuánto se necesita aprender de cada una de ellas para cursarlas con éxito².

Obviamente, en la enseñanza y aprendizaje de los idiomas extranjeros en la universidad han de tenerse en cuenta también estas consideraciones. Estimular el pensamiento para favorecer el dominio de una lengua que no es la propia es una labor docente de gran importancia. De modo más concreto, en el ámbito de los idiomas científico-técnicos existen modalidades y estilos de pensamiento que pueden ayudar a lograr este objetivo.

En el presente artículo se expone una propuesta de trabajo en el aula, en relación con la asignatura denominada Francés Técnico, perteneciente al plan de estudios de la Licenciatura en Enología de la Universidad de La Rioja. Se pretende lograr con ella que los estudiantes vean con claridad hasta dónde llega su dominio de la materia, así como ayudarles a ir consiguiendo un mejor manejo del francés en los campos de la viticultura y la enología y, con ello, una mayor agilidad de pensamiento en la gestión de sus conocimientos acerca de este idioma.

Aunque dicha asignatura pertenece a un plan de estudios universitario actualmente en extinción, las ideas aquí desarrolladas podrían aplicarse y ser de utilidad en la impartición de materias de francés específico recientemente implantados en los nuevos grados.

Este artículo está dirigido sobre todo a docentes en lengua francesa aplicada a los ámbitos científico y técnico. Sin embargo, modestamente se considera que la propuesta que se presenta aquí podría ser válida, como herramienta de trabajo, en la enseñanza de otras lenguas extranjeras dentro de los mencionados campos.

1. "El conocimiento metacognitivo es el conocimiento sobre el conocimiento y el saber, e incluye el conocimiento de las capacidades y limitaciones de los procesos del pensamiento humano, de lo que se puede esperar que sepan los seres humanos en general y de las características de personas específicas -en especial de uno mismo- en cuanto a individuos conocedores y pensantes." (Nickerson, Perkins y Smith, 1987, p. 125).

2. En relación con el concepto de metacognición, Noël (1997, p. 11) menciona y cita a Brown (1978), del cual dice: "Brown a clairement exprimé les différences importantes dans la métacognition notamment quand elle distingue entre savoir quand on sait ('knowing when you know'), savoir ce que l'on sait ('you know what is that you know'), savoir ce que l'on a besoin de connaître ('knowing what you need to know'), connaître l'utilité de stratégies d'intervention ('knowing the utility of active intervention')". Vid. también al respecto Allueva (2002, pp. 69-71). 


\section{LA INVESTIGACIÓN Y LA DOCENCIA EN ESPAÑA SOBRE EL FRANCÉS PARA FINES ESPECÍFICOS}

Se aprecia en los últimos años, en la universidad española, un desarrollo considerable de las investigaciones acerca de la lengua francesa en su utilización en diversos ámbitos especializados. Interesan tanto sus características y especificidades como la manera de abordar su enseñanza en diversas titulaciones universitarias.

Son precisamente las aportaciones realizadas en España las que presentan para el presente trabajo un mayor interés, por cuanto constituyen una referencia más cercana y pueden servir como elementos orientadores más eficaces en el contexto universitario nacional. Esto se considera especialmente importante en aquellas publicaciones en las que se da un enfoque del francés especializado como idioma extranjero, es decir, como materia destinada a la enseñanza ante un alumnado español (FLE).

El francés para fines específicos cubre ámbitos muy variados, que han dado lugar a una producción investigadora igualmente rica y múltiple. De este modo, han ido apareciendo publicaciones de naturaleza lingüística sobre francés y turismo, con aportaciones como las de Maciá Espadas (2005) o Blanco Calvo (2006); o sobre francés jurídico, con trabajos como los de Carvalho (2011) y Giráldez Ceballos-Escalera (2011). También existen aportaciones sobre el francés y el mundo del vino: Sierra Soriano y Mogorrón Huerta (2003); sobre el francés y la publicidad (ámbito empresarial): Cruz Rodríguez (2003), Cubillo Ferreira (2003); o incluso acerca del francés en relación con los deportes: Herráez Pindado (2011).

El enfoque didáctico en la consideración del francés para fines específicos se encuentra en trabajos como los de Cadena Monllor (2004), Olmo Cazevieille (2004), Gómez Ángel (2005), Maciá Espadas (2010), López Santiago (2000 y 2005) y Pisa Cañete (2011), entre otros de publicación más o menos reciente. Los resultados de investigación propuestos se basan en la experiencia docente universitaria de sus autores y están orientados, como es lógico, a su aplicación práctica con el objeto de mejorar la enseñanza del francés especializado. El Marco común europeo de referencia para las lenguas (MCERL), la enseñanza y adquisición de competencias y el aprovechamiento de las nuevas tecnologías son elementos que determinan muchas de estas aportaciones, así como la realidad docente a la que están vinculadas. En nuestra opinión, es indispensable actualmente que el profesorado universitario de idioma extranjero para fines específicos (no sólo de francés) tenga muy presentes estos tres factores en el desarrollo de su labor.

El número 19 de la revista Anales de Filología Francesa de la Universidad de Murcia, correspondiente a 2011, está dedicado al francés para fines específicos. La aportaciones publicadas abordan diversos campos especializados (turístico, gastronómico, jurídico, publicitario, etc.), desde diferentes enfoques (aunque predomina el didáctico).

Para el presente estudio revisten un interés especial, por su mayor proximidad, las aportaciones realizadas en el terreno del francés de la ciencia y la técnica. Sueza Espejo y Torre Montes (2009) y Herráez Pindado (2010) tienen el mérito considerable de establecer, de manera más o menos general, una serie de características propias del discurso científico y técnico en francés. 
Sin embargo, tienen en este artículo una importancia mayor los trabajos de investigación de naturaleza metodológica, en relación con la enseñanza del francés y la importancia de la metacognición. Por ello, merecen ser destacadas las aportaciones de Valiente Jiménez (2004a, 2004b, 2008), que han sido tenidas en cuenta en el presente trabajo, aunque se centren fundamentalmente en la compresión oral del francés. En sus investigaciones están presentes autores como, entre otros, Prieto Sánchez, Weinstein y Meyer, O'Malley, Chamot y Küpper y, de modo más específico Vandergrift y Goh.

\section{AlumNADO Y METACOGNICIÓN}

Al tener cuenta la metacognición de los alumnos, es decir, la conciencia que éstos puedan tener acerca de si saben Francés Técnico (¿sé Francés Técnico?) o de cuánto saben de esta asignatura (¿qué sé sobre Francés Técnico?), hay que partir de la base teórica de que se han matriculado en ella para aprender un idioma con una orientación especializada. Por lo tanto, cabe suponer que ellos mismos conocen las contestaciones a ambas cuestiones, antes de empezar a cursar Francés Técnico, y que las dos son básicamente negativas: no saben nada (o no mucho) de dicha materia.

Por otra parte, para que el alumno llegue a ser consciente de lo que necesita saber sobre la asignatura, es importante la intervención orientadora del profesor, como responsable de la impartición de la misma. Un momento idóneo para que empiece a desarrollarse esta intervención necesaria es el primer día de clase, cuando suele tener lugar la presentación general del curso por parte del docente. Ésta puede ser considerada de por sí como una primera actividad, una primera actuación metacognitiva, para que el alumno, con la ayuda del profesor, llegue a ser capaz de responderse a la pregunta ¿qué necesito saber sobre Francés Técnico? Aquí es importante hacer ver a los estudiantes la distinción entre el francés específico del mundo de la viticultura y la enología (que es, en principio, algo nuevo para ellos) y los contenidos concretos a los que esta lengua especializada da expresión (y con los cuales están familiarizados, por su estudio en otras materias de la titulación de Enología). Esto servirá para que se den cuenta de que no son totalmente desconocedores de la asignatura, de que saben algo (las significaciones). Sin duda ellos son conscientes de esto, pero es muy posible que (por su propia obviedad) no lo tengan suficientemente presente. Podría ser una forma de mostrarles cuáles son los límites de sus saberes en este terreno y hacia dónde tienen que ampliarlos. Otra actuación metacognitiva que puede resultar eficaz sería poner a los alumnos algún tipo de examen de francés genérico el primer día (o en los primeros días). Ellos mismos lo corregirían, siguiendo las indicaciones en clase del profesor. Aquí resultaría de gran utilidad que se cambiaran los exámenes por parejas para corregirlos, y que luego cada uno explicara al otro los fallos que ha tenido. Así también se darían cuenta con mayor claridad de lo que saben, de lo que no saben y de lo que necesitan saber. La realización de esta prueba serviría además al docente para determinar el nivel general de dominio del idioma por parte del alumnado, y para establecer unos objetivos de aprendizaje realistas. Estas dos actuaciones podrían identificarse como realiza- 
ciones concretas de la estrategia metacognitiva ${ }^{3}$ a la que $\mathrm{O}^{\prime}$ Malley y Chamot dan la denominación de planificación ${ }^{4}$.

Hay que tener presente aquí la importancia de la asistencia regular a clase. Esto es algo absolutamente evidente, sin duda. Pero no se debe olvidar que la constancia del trabajo día a día en el aula, con la ayuda del profesor, es una manera tan tradicional como eficaz de hacer que el alumno progrese en el dominio de la materia y llegue a ver con claridad este avance (sobre todo con los errores que cometa y la corrección de los mismos). En este sentido, se genera y se potencia el ejercicio de la metacognición. Por lo tanto, asistir a clase puede ser considerada como una tercera actuación metacognitiva, que encajaría en las estrategias autoadministración y autorregulación, según la taxonomía de O’Malley y Chamot.

Existe la posibilidad de que se produzcan dos errores metacognitivos por parte de los alumnos. En primer lugar, que algunos de ellos piensen que, como ya han estudiado francés anteriormente (en el caso de que así sea), también tienen bastantes conocimientos de Francés Técnico. En segundo lugar (y en sentido contrario), que crean que no saben nada sobre la materia, porque nunca han estudiado francés (o muy poco). En ambos casos podrían estar equivocados sobre el auténtico alcance de lo que saben. Si estas situaciones llegaran a darse entre los estudiantes, las actuaciones metacognitivas expuestas (junto con otras que se verán más adelante) podrían servir para activar en ellos una reflexión que les iría bien para ajustar lo que creen que saben a lo que realmente saben y a partir de ahí identificar bien lo que tendrán que aprender.

\section{Propuesta de trabajo: Objetivos generales}

Como ya se ha anticipado, las finalidades de las actividades que se van a exponer a continuación son dos:

- Ayudar al alumno a desarrollar su capacidad de autoanálisis, en relación con el alcance de sus conocimientos sobre francés específico de los ámbitos vitícola y enológico. Se trata de aportarle una enseñanza adecuada y eficaz sobre cómo utilizar su propio pensamiento al servicio de su beneficio formativo.

- Contribuir a que los conocimientos adquiridos al cursar Francés Técnico lleguen a ser bien asimilados, hasta el punto de poder manejar gramática y vocabulario y traducir de manera rápida, sin dudar ni tener que pararse a pensar en cómo utilizar correctamente dichos conocimientos. Por ello, es importante

3. Doddis y Novoa (1999-2000, pp. 81-82) exponen la definición de estrategia metacognitiva aportada por O'Malley y Chamot: "En la taxonomía de estrategias de aprendizaje de O'Malley y Chamot (1990), se distinguen tres categorías de estrategias: metacognitivas, cognitivas y socioafectivas. Las metacognitivas apelan al conocimiento del sujeto de sus propios procesos cognitivos y cumplen, entonces, una función reguladora del aprendizaje $[\ldots]^{\prime \prime}$.

4. Doddis y Novoa (1999-2000) utilizan, como marco teórico para su trabajo, las mencionadas estrategias de aprendizaje de O'Malley y Chamot, de las cuales reproducen en detalle la relación de estrategias metacognitivas (1999-2000, p. 82). Como se irá viendo, en el presente trabajo se hace alusión a varias de ellas. 
mejorar la agilidad de la memoria, depósito de los conocimientos de idioma extranjero que se van adquiriendo ${ }^{5}$, y también la capacidad para discernir con rapidez cómo utilizar correctamente estos saberes. En consecuencia, en este aprendizaje se deberá llegar a desarrollar una tendencia a que la actividad de pensar, en cuanto reflexión más o menos detenida o profunda, quede reducida al mínimo posible, en favor de una cierta automatización en el manejo de la lengua francesa. Esto será una clara señal de dominio de este idioma. Por el contrario, el titubeo indicará falta de formación al respecto.

Ciertamente, semejante planteamiento puede resultar en principio paradójico, si lo que se pretende es enseñar a pensar al alumno. Pero no es así en absoluto, puesto que la consecución de tal finalidad, al impartir una asignatura de idioma extranjero, no implica anular la actividad mental, sino hacer que ésta sea lo más dinámica y fluida posible. Tampoco existe aquí ninguna contradicción con el hecho de ser consciente de lo que se sabe sobre Francés Técnico y de lo que se tiene que llegar a conocer sobre él. La progresión hacia el manejo del idioma específico con soltura no implica en modo alguno dejar de saber cuánto se ha aprendido y cuánto queda todavía por aprender.

El pensamiento predominante en la impartición y aprendizaje de Francés Técnico ha de ser de tipo convergente ${ }^{6}$. Al tratarse de una asignatura de carácter específico, vinculada al ámbito de la ciencia, el alumno ha de tender a identificar de manera clara y directa la terminología especializada en francés y su equivalente en castellano. Lo mismo puede decirse, básicamente, en lo que concierne a la lectura, comprensión y traducción de textos en francés sobre viticultura y enología, aunque estas labores precisen de más tiempo. En este sentido, el estilo de pensamiento utilizado por el estudiante será básicamente el ejecutivo, consistente en conocer y aplicar correctamente las normas gramaticales propias de la lengua francesa y las correspondencias entre vocabulario especializado del español y del francés ${ }^{7}$, así como en contrastar adecuadamente ambos idiomas.

5. Aquí entrarían en juego dos actividades vinculadas estrechamente con la memoria, como son el almacenamiento y la recuperación: "Los estudiosos de la memoria distinguen, asimismo, entre actividades de almacenamiento y de recuperación. Como sus propios nombres indican, las actividades de almacenamiento sitúan información en la memoria mientras que las actividades de recuperación recogen información de la memoria. Almacenar significa atender, codificar, memorizar, estudiar y cosas por el estilo; 'aprender' suele ser un buen sinónimo. Recuperar significa reconocer, recordar, reconstruir el 'recuerdo' de lo que se ha almacenado anteriormente" (Flavell, 1984, p. 203).

6. "Pensamiento convergente es el que busca una única solución a un problema, la más apta, apropiada o correcta. Es un pensamiento lógico, vertical, analítico, deductivo, riguroso, selectivo, con una consecución de fases, formal y crítico" (Allueva, 2007, p. 138). Vid. también Sternberg (1999, pp. 210-211).

7. "A las personas ejecutivas les gusta seguir reglas y prefieren los problemas estructurados y planteados de antemano. Les gusta rellenar las lagunas de estructuras ya existentes en vez de crear estructuras ellas mismas. Algunas de las actividades que suelen preferir son resolver problemas matemáticos ya dados, aplicar reglas a problemas, dar conferencias o impartir clases basadas en ideas ajenas, y hacer cumplir normas" (Sternberg, 1999, p. 41). 


\section{Propuesta de trabajo: actividades}

\section{Actividades a realizar}

Se plantean aquí bloques fundamentales de ejercicios (ver anexo 2):
A) Francés genérico
- Nivel elemental: trabajo con ejercicios de gramática, vocabulario, conjuga- ción de verbos y traducción de frases cortas.
- Nivel avanzado: explotación gramatical de un texto genérico o especializado.

B) Léxico específico

- Trabajo con imágenes relativas a viticultura, vinificación y cata. Hay que añadirles la terminología específica en francés sobre las labores, procesos y elementos que se muestran en ellas, expresadas en castellano.

- Trabajo de explotación de textos especializados.

C) Traducción. Trabajo consistente en traducir del francés al castellano textos breves pertenecientes a los ámbitos de la viticultura, la vinificación y la cata.

Por lo general, los estudiantes, debido quizás a una trayectoria formativa en idiomas extranjeros orientada (desde muy pronto) de forma preferente al inglés, no suelen mostrar un dominio demasiado profundo de la lengua francesa. Esto hace necesario que el planteamiento de los ejercicios sea relativamente simple, en especial los de francés genérico y los que consisten en trabajar con textos. De todas formas, los libros y artículos científico-técnicos en francés no suelen caracterizarse por el uso de un estilo demasiado complicado. Con todo, siempre es posible que el nivel general del alumnado varíe de un curso a otro. Esto refuerza la necesidad de la realización (antes señalada) de un examen de francés genérico al comienzo de curso, con una finalidad informativa. Por ello, es conveniente prever ejercicios con niveles de dificultad diferente. Esto ha llevado a plantear, en el apartado de francés genérico, dos clases de actividades, una de nivel elemental y otra de nivel más avanzado (ver anexo 2). El profesor ofrecerá a los estudiantes una u otra, en función del dominio general del idioma que éstos hayan demostrado tener en clase. Por supuesto, puede establecerse un número mayor de niveles y proponer ejercicios adecuados para cada uno de ellos. Se dispondría así de un abanico de actividades de francés genérico más amplio y más flexible y adaptable a los estudiantes.

\section{Objetivos específicos de cada actividad}

Los objetivos propios de cada una de las distintas actividades se subordinan a las finalidades generales que acaban de ser expuestas. Son los siguientes:

- Alcanzar un conocimiento y un manejo adecuados del francés genérico, mediante la correcta realización de los ejercicios del bloque A.

- Alcanzar un conocimiento y un manejo adecuados del francés específico de la viticultura y la enología, mediante la correcta realización de los ejercicios de los bloques B y C. Se da aquí una importancia especial a la comparación entre el francés y el castellano especializados. 
Desarrollo-aplicación de cada actividad

En principio, se ha considerado que la realización de las actividades ha de llevarse a cabo una vez que hayan transcurrido cinco o seis semanas, aproximadamente, desde el comienzo de curso. De este modo, los alumnos ya habrán recibido un volumen mínimo razonable de enseñanzas de Francés Técnico en los tres aspectos establecidos, cuya asimilación podrá ser objeto de estudio y valoración.

Asimismo, se ha estimado conveniente disponer de una semana de trabajo en el aula para Ilevarlas a cabo. Esto equivale a dedicar tres horas a su realización, a razón de una hora por cada una de ellas. En la primera hora se entregaría a cada alumno una serie de ejercicios de francés genérico (bloque A); en la segunda hora una imagen o un texto con vocabulario específico (bloque B), y en la tercera un pequeño texto en francés especializado para traducir (bloque C).

\section{Evaluación de cada actividad}

Se proponen dos formas de evaluación, no necesariamente excluyentes entre sí:

- Corrección y evaluación de las actividades por el profesor fuera del aula. Aquí el trabajo de evaluación recae por entero en el docente, que deberá indicar a los estudiantes en clases posteriores las equivocaciones cometidas y la manera de corregirlas. Se iniciaría así un proceso de realimentación necesario para potenciar la metacognición.

- Corrección en el aula, realizada entre profesor y alumnado, y comprobación por cada estudiante de sus aciertos y errores. Se llevaría a cabo en la semana siguiente a la realización de las actividades. La labor de evaluación es compartida por profesor y estudiantes. Éstos podrán valorar su trabajo de forma más directa y personalizada. Por lo tanto, hay aquí, al menos en cierta medida, un trabajo de autoevaluación muy importante también para el desarrollo de la metacognición.

Estas correcciones guardarían un cierto parecido con las estrategias metacognitivas de evaluación que Valiente Jiménez (2004a: 131 y 254-258) propone en la realización de pruebas de comprensión oral. Forman parte del conjunto de distintas estrategias metacognitivas que esta investigadora ha estudiado en su aplicación a este tipo de pruebas (Valiente Jiménez: 2004a: 228-258). Se basa en la clasificación de las estrategias de compresión oral propuestas por Vandergrift (Valiente Jiménez: 2004a: 129-136).

\section{Realimentación y refuerzo a los alumnos}

En las distintas de actividades propuestas, se considera que lo más adecuado es desarrollar un trabajo de corrección, iniciado en el aula (con los procedimientos de evaluación que se acaban de indicar) y continuado por el alumnado fuera de ella (de manera autónoma). Habría que dedicar especial atención a aquellos errores que hubieran sido cometidos con mayor frecuencia por el conjunto de los estudiantes, pues podrían ser indicativos de equivocaciones del profesor en su labor docente. En tal caso, éste tendría que proceder también a una necesaria autoevaluación. El repa- 
so posterior en clase sería una labor imprescindible para los estudiantes, aunque se corriera el riesgo de hacer que la enseñanza se ralentizara. Esto sería asumible, si con ello se consigue mantener la calidad de la docencia y garantizar el desarrollo de un buen proceso de aprendizaje. Consistiría en la realización reiterada de ejercicios de la misma naturaleza que los inicialmente efectuados.

El trabajo independiente del alumno se considera igualmente indispensable, ya que éste se enfrentaría en soledad (o con otros compañeros, trabajando en equipo) a la labor de práctica del idioma específico. Tendría que pensar de modo diferente a como posiblemente piensa en el aula, donde espera la ayuda del docente. Al carecer de ésta, posiblemente se haría aún más consciente que en clase de lo que sabe, de lo que no sabe y de lo que tiene que saber. Aquí cobrarían una enorme importancia la autoevaluación y la corrección autónoma (sin que se excluya totalmente el recurso al docente, en el aula o en tutorías, si fuera preciso). El manejo del aula virtual y de diferentes páginas Web, con ejercicios de francés genérico y específico (que disponen de una corrección automática) sería muy necesario.

Tanto la corrección en el aula (en parte) como la labor autónoma del estudiante podrían considerarse como aplicaciones concretas de la estrategia metacognitiva de O'Malley y Chamot denominada autoevaluación.

En la evaluación, autoevaluación y la realimentación se aprecia también una cierta aplicación de la estrategia metacognitiva de identificación de problemas, que consistiría en "identificar explícitamente el punto central que necesita solución en una tarea o identificar un aspecto de la tarea que dificulta completarla con éxito" (Valiente Jiménez, 2004a: 132). Se trataría, obviamente, de detectar dificultades que no se han sabido resolver durante la realización de las actividades, al objeto de poder superarlas en ejercicios que se efectúen más adelante.

Posteriormente, y al objeto de comprobar la progresión del alumnado, se le podría proponer a éste la realización de una segunda serie de actividades del mismo tipo y con idéntica planificación y desarrollo que las de la primera serie, aunque con un nivel de dificultad superior. Ha de entenderse por progresión la capacidad del estudiante para asimilar las correcciones de los errores cometidos en la primera serie, el mantenimiento de los aciertos (no olvidar lo aprendido) y el aprendizaje de nuevos contenidos de Francés Técnico.

Esta segunda serie podría llevarse a cabo cinco o seis semanas después de la primera serie, tiempo mínimo suficiente para haber podido impartir una cantidad aceptable de nuevas enseñanzas. Por otra parte, se realizaría con bastante anticipación con respecto al término del curso. Así se podría efectuar también una segunda realimentación con vistas a la evaluación final.

Se ofrecería al alumno una cantidad mayor de ejercicios de francés genérico y de imágenes y de textos específicos. El bloque de actividades comprendería las ya abordadas en la primera serie más otras nuevas. Obviamente, se le daría más tiempo para efectuarlas (una hora por cada una de ellas). De este modo, se podría valorar la progresión de los estudiantes, tal como ha sido definida más arriba, y también comprobar si la tendencia a la automatización en el empleo del francés y la rapidez del pensamiento convergente y ejecutivo se van desarrollando (o no) correctamente. 
También se podría proponer a los alumnos que efectuasen un Juicio Metacognitivo (ver anexo 1). Consistiría en pasarles dos pequeños cuestionarios. Uno se contestaría antes de la realización de los ejercicios (aunque después de haberlos revisado), y el otro justo después (aunque antes de proceder a su corrección). El primero contendría preguntas acerca de lo que ellos creen saber y de lo que consideran que precisan saber sobre Francés Técnico para resolver bien las actividades que se les propone. En el segundo se les preguntaría, básicamente, si piensan que tenían suficientes conocimientos de la materia para poder hacer bien lo que se les ha pedido Se darían aquí otras dos actuaciones metacognitivas. Una encajaría con la estrategia planificación y la otra con la estrategia autoevaluación, dentro de la taxonomía de O'Malley y Chamot.

De modo más concreto, y en relación con la enseñanza de un idioma extranjero, la realización del primer cuestionario se correspondería (en cierto modo) con el desarrollo de las estrategias metacognitivas de planificación de Valiente Jiménez (2004a: 129-131 y 229-242). A la vista de los ejercicios, y en función de la mayor o menor capacidad que crea tener para resolverlos, el estudiante prepararía y abordaría de una u otra forma su realización. En este sentido, el primer cuestionario tendría un cierto valor informativo de naturaleza prospectiva (Valiente Jiménez, 2004b). La cumplimentación del segundo cuestionario se aproximaría (también en cierta medida) a las estrategias metacognitivas de evaluación de Valiente Jiménez (2004a: 131 y 254-258) y tendría un valor informativo retrospectivo (Valiente Jiménez, 2008).

Planificación y autoevaluación permitirían a los estudiantes conocerse mejor y ser conscientes de si sus apreciaciones sobre su conocimiento de Francés Técnico en distintos momentos y actividades son optimistas, pesimistas o ajustadas a la realidad. En consecuencia, les ayudarían a ser realistas y a saber caer verdaderamente en la cuenta de lo que saben, de lo que no saben, de lo que necesitan saber y de qué medios deben poner en marcha para llegar a adquirir los conocimientos que les falten.

\section{IMPLEMENTACIÓN DE LAS ACTIVIDADES Y VALORACIÓN DE LOS RESULTADOS}

Las dos series de actividades mencionadas han sido puestas en práctica con la asignatura Francés Técnico una sola vez, hasta el momento actual. La primera serie se pasó a los alumnos sin previo aviso, como una práctica más de clase. Al tratarse de una serie de ejercicios sobre todos los aspectos de la asignatura y sobre la materia impartida hasta ese momento, podía decirse que se estaba haciendo una especie de simulacro de examen. En cierto modo, se trataba de un "examen sorpresa" (sin ninguna trascendencia para la calificación final) que permitiría ver el estado real de los conocimientos de los estudiantes. También se les pasó unos cuestionarios para que realizasen su Juicio Metacognitivo.

La valoración de los resultados obtenidos por los alumnos llevó a apreciar un nivel de acierto bastante bajo en la mayoría de los casos. Por lo tanto, lo imprevisto de esta primera serie permitió ver que, de modo general, no había predominado entre los estudiantes una actitud de trabajo más o menos continuado con la asignatura. Además, no deja de ser revelador, en consonancia con esto, que la revisión del Juicio Metacognitivo realizado antes de abordar las actividades Ilevase a apreciar 
que la mayor parte de ellos no estuviesen seguros de su capacidad ni de sus conocimientos para realizarlas bien. Demostraron, por lo tanto, un buen nivel de madurez en lo que podría llamarse una autocrítica negativa anticipada sobre sus conocimientos de Francés Técnico.

En cualquier caso, la escasa eficacia en la realización de esta primera serie de actividades indicaba poco dominio de la lengua francesa específica. Por lo tanto, aún no se había alcanzado en ese momento del cuatrimestre un tipo de pensamiento convergente, de estilo ejecutivo y que discurriese con agilidad.

La segunda serie de actividades fue anunciada a los alumnos al término de la primera. Por lo tanto, se les avisó con antelación suficiente. Es probable que esto, junto con el acertado (y negativo) Juicio Metacognitivo, les hubiera impulsado a trabajar en los contenidos de Francés Técnico con mayor insistencia y constancia: los resultados ofrecidos en esta serie fueron claramente mejores que los de la anterior, sobre todo en francés genérico y traducción. En el apartado de léxico se observó un nivel de errores algo inferior. La mejora en este terreno no fue muy notable. Esto permitió establecer que el vocabulario es el aspecto de la asignatura que les resultó más difícil. Parece claro que, dada su abundancia y variedad, el esfuerzo de memoria y asimilación necesario para conocerlo y dominarlo fue muy considerable, incluso mayor que para los otros dos aspectos.

De todos modos, el mayor acierto general en la realización de esta segunda serie reflejó un avance no desdeñable en la mencionada tendencia hacia la automatización en el empleo del francés y hacia el ejercicio del pensamiento convergente y ejecutivo.

Es importante señalar que las actividades que se pasó a los alumnos tenían básicamente la misma organización en tres bloques ( $A, B, y$ ) que se ha expuesto anteriormente. Sin embargo, presentaban ciertas diferencias con respecto a lo que aquí se ha explicado y que se puede ver más detalladamente en el anexo 2. En el apartado de francés genérico sólo se les dio ejercicios de nivel elemental. En la parte de vocabulario únicamente se les proporcionó imágenes. El texto para traducción que aquí se ha incluido también es distinto de los que se les entregó a ellos, aunque presenta un nivel de dificultad semejante. El diseño definitivo de las actividades descrito más arriba y ejemplificado en el anexo 2 es un modelo, producto de reflexiones posteriores a esta implementación e incluye ciertas modificaciones y mejoras. Éstas son la distinción de dos tipos de ejercicios, de diferente nivel (básico y avanzado), para francés genérico; la inclusión de algún texto en el apartado de vocabulario, con el objeto de que los estudiantes Ileguen a conocer y manejar el léxico insertado en el discurso técnico y no sólo aisladamente; en cuanto a la traducción, se ha preferido proponer aquí un texto procedente de una publicación especializada aparecida más recientemente.

\section{CONCLUSIONES}

La propuesta de trabajo docente en el aula expuesta aquí ha llevado a establecer varias conclusiones de interés.

Dicha propuesta tiene una dimensión informativa que cabría considerar como valiosa, por cuanto permitiría al estudiante lograr un mayor conocimiento de sí 
mismo, en lo que concierne a sus limitaciones y posibilidades de mejora en el dominio del francés especializado. Esto posibilitaría un siempre necesario ejercicio de autoevaluación a lo largo del curso, una gestión más eficaz del trabajo de aprendizaje. Por lo tanto, supondría un desarrollo de habilidades metacognitivas. En este sentido, no se ha de olvidar el hecho de que actualmente las asignaturas de idioma extranjero científico-técnico (no sólo las de francés) son habitualmente optativas dentro de los planes de estudios de las titulaciones universitarias a las que pertenecen. Esto implica que los estudiantes tienden a darles menos importancia que a las materias obligatorias. Con lo cual, van a dedicarles, previsiblemente, menos tiempo y trabajo. Se incurre así en el riesgo de que el ejercicio de la metacognición quede relegado. Las actividades propuestas sirven para tratar de evitar (o, al menos, reducir) este peligro. La diferencia de rendimiento del alumnado entre la primera serie y la segunda, constatada en su implementación, así lo demuestra.

Además, sería conveniente, desde el comienzo del curso, ir introduciendo al alumnado, aunque fuese de forma somera, a un cierto conocimiento acerca de estrategias metacognitivas. No se trataría solamente de llevar a cabo la labor orientadora del profesor con la presentación general de la asignatura el primer día de clase, o de poner a los estudiantes algún examen o prueba informativa al inicio del curso. También habría que hablarles acerca de cómo planificar la realización de ejercicios y exámenes y de cómo evaluar los resultados de los mismos. También se les podría informar sobre cómo supervisar y corregir su elaboración mientras la están llevando a cabo, aplicando estrategias metacognitivas de monitorización (Valiente Jiménez, 2004: 131 y 243-253). Esto constituiría una mejora en relación con la implementación de las actividades expuesta aquí.

Es importante ajustar el grado de dificultad de las actividades, sobre todo las de francés genérico, al nivel de dominio real de los alumnos. Éste puede variar de un año para otro, y por ello hay que prever diversos bloques de ejercicios, de mayor y menor complicación. Cabría pensar, en principio, que en la enseñanza de asignaturas de inglés específico esta cuestión tendría menos importancia que en francés. Se supone que los estudiantes conocen mejor la lengua inglesa, puesto que la han estudiado durante más tiempo (desde la Educación Primaria). Aun así, no deja de ser aconsejable que el docente de idioma extranjero científico-técnico se adapte inicialmente al nivel del alumnado, para luego ayudarles a progresar en su dominio de la lengua en cuestión.

Esta propuesta de trabajo en el aula podría tener también una utilidad formativa especialmente interesante para el alumno. Mejoraría sus conocimientos sobre francés específico y accedería a un manejo más rápido y automatizado de los mismos. Por lo tanto, el pensamiento convergente y el estilo de pensamiento ejecutivo actuarían con mayor agilidad. Además, como se ha señalado, la adquisición de una mayor soltura en el uso del idioma no supone en absoluto dejar de ser consciente de lo que se sabe, de lo que se ha progresado y de lo que aún se ignora y se debe aprender.

Las actividades presentadas han sido concebidas para su realización en el aula, pero no dejan por ello de tener una proyección fuera de ella. Existe, como se ha podido ver, la necesidad de llevar a cabo un trabajo autónomo (individualmente 
o en grupo), que aumentaría notablemente su eficacia. La presencia del alumno como protagonista de su propia iniciativa de aprendizaje mantiene aquí su importancia.

Por último, no deja de ser llamativo que la experiencia aportada por la puesta en práctica de las dos series de actividades haya demostrado que una herramienta esencial para acceder a un mejor desarrollo de todos los valores mencionados (metacognición, dominio del idioma, pensamiento convergente y ejecutivo, autonomía en el aprendizaje) sea algo tan antiguo, conocido y bien valorado (aunque no siempre bien practicado) como la perseverancia y la continuidad en el trabajo por parte del alumno. Parece claro que las tentativas actuales de innovación educativa no tienen por qué ser incompatibles con el mantenimiento de prácticas y virtudes tradicionales que desde siempre se han mostrado eficaces y productivas.

\section{REFERENCIAS BIBLIOGRÁFICAS}

ALLUEVA, P. (2002). Desarrollo de habilidades metacognitivas: programa de intervención. [Zaragoza]: Consejería de Educación y Ciencia.

ALLUEVA, P. (2003). Realización de Programas para el Desarrollo de las Habilidades Metacognitivas. Revista Argentina de Psicopedagogía, 57, 1-5.

ALLUEVA, P. (2007). Habilidades del pensamiento. En M. Liesa Orús, P. Allueva Torres y M. Puyuelo Simelio (Eds.), Educación y acceso a la vida adulta de personas con discapacidad (pp. 133-149). [Huesca]: Fundación Ramón J. Sender.

BLANCO CALVO, P. (2006). Aproximación nocional, formal y semántica del vocabulario del francés del turismo. En D. Bonnet, $M^{a}$. J. Chaves García y N. Duchêne (Eds.), Littérature, Langage et Arts: Rencontres et Création. Huelva: Servicio de Publicaciones de la Universidad de Huelva.

BROWN, A. L. (1978). Knowing when, where and how to remember: a problem of metacognition. En R. Glaser (Ed.), Advances in Instructional Psychology. Vol. 1. Hillsdale, N. J.: Lawrence Erlbaum Associates.

CADENA MONLLOR, Ma․ L. (2004). (Un) útil para los estudios turísticos. Reflexiones didácticas y diseño de un programa para la E. U. de Estudios Empresariales de Huesca. Revista de Gestión Pública y Privada, 9, 63-70.

CARVALHO, C. (2011). Simplification du Français Juridique: Étude appliquée à la proposition de loi du 22 juillet 2008 relative à la «Simplification et clarification du droit et à l'allègement des procédures». En M. Sanz y J. Verdegal (Eds.), Construcción de identidades y cultura del debate en los estudios en lengua francesa [recurso electrónico] (pp. 136-149). Castelló de la Plana: Publicacions de la Universitat Jaume I.

CRUZ RODRÍGUEZ, J. M. (2003). Le slogan publicitaire: une lecture des notions pour la vente des produits et des services dans les secteurs du transport aérien et de I'hôtellerie. En Mํ. J. Salinero Cascante e I. Iñarrea Las Heras (Eds.), El texto como encrucijada: estudios franceses y francófonos. 2 vols. (vol. 2, pp. 345-356). Logroño: Universidad de La Rioja. Servicio de Publicaciones. 
CUBILLO FERREIRA, C. M. (2003). Le slogan publicitaire: une lecture des notions pour la vente des produits et des services automobiles. En $M^{a}$. J. Salinero Cascante e I. Iñarrea Las Heras (Eds.), El texto como encrucijada: estudios franceses y francófonos. 2 vols. (vol. 2, pp. 357-366). Logroño: Universidad de La Rioja. Servicio de Publicaciones.

DODDIS, A. y NOVOA, P. (1999-2000). Estrategias de aprendizaje metacognitivas en la comprensión de lectura en inglés como segunda lengua: un estudio de casos. Lenguas Modernas, 26-27, 79-93.

FLAVELL, J. H. (1993). El desarrollo cognitivo. Madrid: Visor.

GIRÁLDEZ CEBALLOS-ESCALERA, J. (2011). Analyse du discours juridique: du «droit dur» au «droit souple». En M. Sanz y J. Verdegal (Eds.), Construcción de identidades y cultura del debate en los estudios en lengua francesa [recurso electrónico] (pp. 179-191). Castelló de la Plana: Publicacions de la Universitat Jaume I.

GÓMEZ ÁNGEL, B. (2005). L'espace virtuel de la classe en français langue de spécialité. En Á. Sirvent Ramos (Ed.), Espacio y Texto en la cultura francesa / Espace et Texte dans la culture française. 3 vols. (vol. 3, pp. 1.849-1.857). Alicante: Servicio de Publicaciones de la Universidad de Alicante.

HERRÁEZ PINDADO, J. (2010). La enseñanza del francés técnico en la Universidad Politécnica de Madrid. En La culture de l'autre: l'enseignement des langues à I'Université. Lyon: La clé des langues. Disponible en: http://dialnet.unirioja.es/ descarga/articulo/4027828.pdf.

HERRÁEZ PINDADO, J. (2011). El vocabulario del boxeo como fuente de metáforas violentas en el deporte. En M. Sanz y J. Verdegal (Eds.), Construcción de identidades y cultura del debate en los estudios en lengua francesa [recurso electrónico] (pp. 217-227). Castelló de la Plana: Publicacions de la Universitat Jaume I.

LÓPEZ SANTIAGO, M. (2000). Enseñanza del francés técnico para arquitectos. Explotación didáctica: estudio del vocabulario técnico o cómo perder la cabeza por la arquitectura. En M. Serrano Mañes, L. Avendaño Anguita y Ma. C. Molina Romero (Eds.), La philologie française à la croisée de I'an 2000. Panorama linguistique et littéraire. 2 vols. (vol. 2, pp. 223-231). Granada: Asociación de Profesores de Filología Francesa de la Universidad Española.

LÓPEZ SANTIAGO, M. (2005). El diccionario en la enseñanza del francés para fines específicos. En Á. Sirvent Ramos (Ed.), Espacio y Texto en la cultura francesa / Espace et Texte dans la culture française. 3 vols. (vol. 3, pp. 1859-1868). Alicante: Servicio de Publicaciones de la Universidad de Alicante.

MACIÁ ESPADAS, N. (2005). Le français comme langue spécifique: le français du tourisme. En Á. Sirvent Ramos (Ed.), Espacio y Texto en la cultura francesa / Espace et Texte dans la culture française. 3 vols. (vol. 3, pp. 1869-1877). Alicante: Servicio de Publicaciones de la Universidad de Alicante.

MACIÁ ESPADAS, N. (2010). Étudier le français du tourisme à l'aide des TICE. En La culture de l'autre: I'enseignement des langues à l'Université. Lyon: La clé des langues. Disponible en: http://dialnet.unirioja.es/descarga/articulo/4027869.pdf.

NICKERSON, R. S.; PERKINS, D. N. y SMITH, E. E. (1987). Enseñar a pensar: aspectos de la aptitud intelectual. Barcelona: Paidós. 
NOËL, B. (1997). La métacognition. París, Bruselas: De Boeck Université.

OLMO CAZEVIEILLE, F. T. (2004). Intégration des nouvelles stratégies d'enseignement dans un cours de français sur la production avicole. En Suso López, J. y López Carrillo, R. (Eds.), Le français face aux défis actuels. Histoire, langue et culture. 2 vols. (vol. 2, pp. 767-774). Granada: Universidad de Granada, APFUE, GILEC.

O'MALLEY, J. M. y CHAMOT, A. U. (1990). Learning strategies in second language acquisition. Cambridge: Cambridge University Press.

PISA CAÑETE, Mán. T. (2011). Les TIC et I'apprentissage d'une langue-culture dans des cours de français avec des objectifs spécifiques. En M. Sanz y J. Verdegal (Eds.), Construcción de identidades y cultura del debate en los estudios en lengua francesa [recurso electrónico] (pp. 566-578). Castelló de la Plana: Publicacions de la Universitat Jaume I.

REYNIER, A. (2000). Manuel de viticulture. Guide technique du viticulteur. Londres, Nueva York, París: Technique \& Documentation.

RIBEREAU-GAYON, P. et al. (1975-1977). Traité d'œnologie: sciences et techniques du vin. 4 vols. París: Dunod.

SANSOT, TH.; SALMON, C. y MULINAZZI, W. (2011). Clarification par système de flottation novateur. Revue Française d'CEnologie, 245, 10-14.

SIERRA SORIANO, A. y MOGORRÓN HUERTA, P. (2003). Quels termes utiliser pour parler du vin? Étude contrastive français-espagnol. En $M^{a}$. J. Salinero Cascante e I. Iñarrea Las Heras (Eds.), El texto como encrucijada: estudios franceses y francófonos. 2 vols. (vol. 2, pp. 591-603). Logroño: Universidad de La Rioja. Servicio de Publicaciones. Disponible en: http://dialnet.unirioja.es/descarga/ articulo/1011620.pdf.

STERNBERG, R. J. (1999). Estilos de pensamiento: claves para identificar nuestro modo de pensar y enriquecer nuestra capacidad de reflexión. Barcelona: Paidós.

SUEZA ESPEJO, Ma․ J. y TORRE MONTES, Ma․ L. (2009). El francés como lengua de comunicación científica para docentes universitarios. En À. Santa y C. Solé Castells (Eds.), Texto y sociedad en las letras francesas y francófonas (pp. 979-990). Lleida: Departament de Filologia Clàssica, Francesa i Hispànica. Universitat de Lleida. Disponible en: http://dialnet.unirioja.es/descarga/libro/460231.pdf.

VALIENTE JIMÉNEZ, Mã . J. (2004a). Estrategias de enseñanza-aprendizaje en la comprensión oral del francés como segunda lengua en la universidad. Madrid: Universidad Complutense de Madrid. Disponible en: http://eprints.ucm.es/tesis/edu/ ucm-t28034.pdf.

VALIENTE JIMÉNEZ, Ma․ J. (2004b). Uso de la estrategia metacognitiva de planificación en la comprensión oral de una lengua extranjera. En A. M. Suárez, F. RamaIlo y X. P. Rodríguez Yáñez (Eds.). Bilingual Socialization and Bilingual Language Acquisition: Proceedings from the Second International Symposium on Bilingualism. (pp. 781-792). Vigo: Servicio de Publicaciones de la Universidad de Vigo.

VALIENTE JIMÉNEZ, Ma․ J. (2008). Régulation métacognitive du contrôle de la compréhensión orale en FLE. Porta Linguarum, 9, 79-81. 


\section{ANEXOS}

\section{Ejemplos de cuestionarios para el Juicio Metacognitivo ${ }^{8}$ :}

- Inmediatamente anterior a la realización de las actividades:

Contesta las siguientes preguntas en el orden en que se exponen, poniendo una cruz junto a la respuesta que elijas:

1. ¿Crees que serás capaz de resolver correctamente los ejercicios de francés específico propuestos?

\begin{tabular}{|l|l|l|}
\hline Sí & No & No sé \\
\hline
\end{tabular}

2. ¿Crees que tienes conocimientos necesarios para resolver correctamente los ejercicios de francés específico propuestos?

\begin{tabular}{|l|l|l|}
\hline Sí & No & No sé \\
\hline
\end{tabular}

3. ¿Qué conocimientos crees que te faltan para resolver correctamente esta clase de ejercicios?

- Inmediatamente posterior a la realización de las actividades:

Contesta las siguientes preguntas, en el orden en que se exponen, poniendo una cruz junto a la respuesta que elijas:

1. ¿Crees que has sido capaz de resolver correctamente los ejercicios de francés específico propuestos?

\begin{tabular}{|l|l|l|}
\hline Sí & No & No sé \\
\hline
\end{tabular}

2. ¿Crees que tenías los conocimientos necesarios para resolver correctamente los ejercicios de francés específico propuestos?

\begin{tabular}{|l|l|l|}
\hline Sí & No & No sé \\
\hline
\end{tabular}

3. ¿Qué conocimientos crees que te faltan para resolver correctamente esta clase de ejercicios?

8. Estos cuestionarios son una adaptación al presente artículo de los publicados originalmente en Allueva (2003, pp. 3-4). 


\section{Ejemplos de actividades':}

A) Francés genérico.

Nivel elemental. Ejercicios:

- Completar las frases siguientes con la forma verbal conveniente, conjugada en presente de indicativo:

Elle (adorer) Paris.

Vous (habiter) en Allemagne.

Tu (s'appeler) Pierre.

Je (vouloir) danser.

Ils (préférer) la montagne.

- Traducir al francés:

¿Cómo te llamas?

Se habla español.

Sylvie hace deporte.

¿Qué es eso?

\section{Vivimos en Madrid.}

- Poner junto a cada sustantivo el artículo determinado que le corresponda:

..... rameau, ..... racine, ..... sarment, ..... éraflage, ..... cuves,

..... greffe, ..... greffages, ..... cep, ..... vrille, ..... mise en bouteille.

- Completar las frases siguientes poniendo el adjetivo entre paréntesis en el género conveniente:

9. Como queda indicado, las actividades que aquí se exponen son solamente ejemplos. Tienen una finalidad fundamentalmente ilustrativa y orientativa. Por lo tanto (y como es obvio), no constituyen en modo alguno los únicos (ni los mejores) tipos de ejercicios que pueden realizarse para el desarrollo de la presente propuesta de trabajo en el aula. Cada docente podrá elegir o preparar las actividades (tanto de ámbito genérico como especializado) que mejor se adecuen, por su nivel o su naturaleza, a las características propias de la asignatura de idioma específico que imparta. 
Carmen est (chanteur)

Walter est (allemand)

Valérie est (musicien)

Anne est (étudiant)

James est (américain)

- Transformar las siguientes frases afirmativas en frases negativas:

Nous avons les cadeaux.

Elles écrivent des romans.

II aime la musique classique.

Tu regardes les photos.

Vous connaissez la secrétaire.

Nivel aVANZADO. TeXto:

Règles pratiques de la dégustation.

On doit tenir compte d'un certain nombre de règles concernant le mode opératoire de la dégustation. Si l'on doit goûter une série de vins, on commence par les plus légers [...]. On déguste les vins rouges avant les vins blancs [...]. Les vins jeunes se goûtent avant les vins vieux. [...]

Les échantillons ne doivent être ni trop chauds, ni trop froids et, bien entendu, pour que des comparaisons soient possibles, ils doivent être tous à la même température. II est bon avant dégustation de les placer quelques heures dans un local à la température voulue. [...]

Pour éviter de goûter des vins plus ou moins mâchés par l'effet de l'oxygène, ils doivent être goûtés aussitôt après le prélèvement de l'échantillon ou l'ouverture de la bouteille, ou si la dégustation est retardée on les garde quelques jours en repos à l'abri de I'air en flacons bien bouchés. [...]

Il est souvent plus intéressant de goûter et de comparer des vins "nature" que des vins de coupage, dans lesquels les caractères sont atténués et qui tendent vers 
quelques types assez uniformes, sans défaut, mais souvent sans caractère, sans personnalité. [...]

Par la diversité des types, la gamme des qualités, la dégustation des vins fins est évidemment à la fois la plus difficile et la plus intéressante. Pour les vins courants on se contente d'une certaine neutralité, d'une uniformité, de l'absence de défauts; il y a ainsi des vins sans personnalité, qu'il est impossible de décrire.

(Ribéreau-Gayon, et al., 1975-1977: 46-47).

\section{Cuestiones:}

1. Subrayar en azul las frases con una proposición subordinada de finalidad.

2. Subrayar en negro las frases con una proposición subordinada condicional.

3. Subrayar en rojo los verbos en forma impersonal.

4. Poner la frase siguiente en forma negativa: Les vins jeunes se goûtent avant les vins vieux.

5. Poner el verbo de la frase siguiente en futuro: Les échantillons ne doivent être ni trop chauds ni trop froids.

6. Poner el verbo de la frase siguiente en passé composé: Pour les vins courants on se contente d'une certaine neutralité, d'une uniformité, de l'absence de défauts.

7. Escribir las proposiciones subordinadas de relativo, subrayar los pronombres relativos que contengan e identificar sus respectivos antecedentes.

8. Escribir los adjetivos que aparecen en el texto en grado superlativo relativo.

9. Dar un sinónimo de los términos siguientes: abri, flacon, bouché, défaut, courant, neutralité. El sinónimo puede extraerse del texto, aunque no en todos los casos.

10. Traducir al castellano las frases siguientes:

On déguste les vins rouges avant les vins blancs.

... ils doivent être goûtés aussitôt après le prélèvement de l'échantillon.

... on les garde quelques jours en repos à l'abri de l'air en flacons bien bouchés. 
B) Vocabulario específico.

- Añadir la palabra o expresión francesa equivalente a cada uno de los términos o expresiones en castellano que aparecen en la imagen siguiente:

\section{VINIFICACIÓN DEL VINO TINTO}

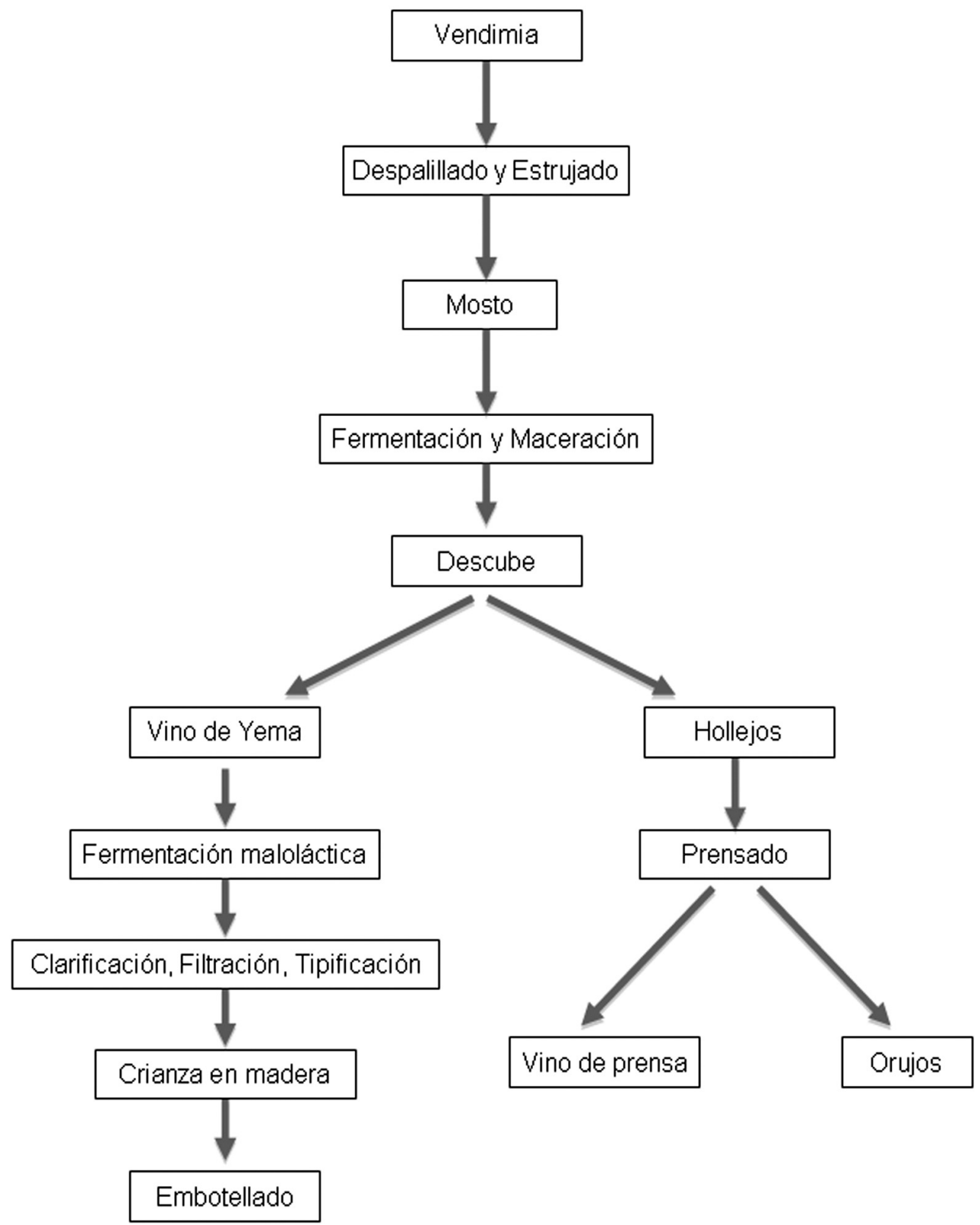


- Completar el texto siguiente con las palabras que se proponen: teneur, réduction, alcoolique, gustative, arômes, fruité, esters, moûts, débourbage, lourds.

Il y a longtemps que les vinificateurs sont convaincus de l'amélioration des vins lorsqu'ils résultent d'un ............ efficace des moûts correspondants. Si la ........... en bourbes est élevée en début de fermentation les vins auront des goûts végétaux, des arômes ............ des bouches amères. Ils seront plus sensibles à l'oxydation [...] et plus colorés. En fin de fermentation, des défauts de ........... sont observés et difficiles à éliminer, même après une mise au propre et des aérations répétées. Les vins issus de .......... bien clarifiés ont, au contraire, un caractère ........... net et stable. Leurs teneurs en alcools supérieurs qui confèrent de la lourdeur sont faibles et ils sont plus riches en acétates d'alcools supérieurs et en éthyliques d'acides gras, aux positifs.

(Sansot, Salmon et Mulinazzi, 2011: p. 10).

C) Traducción. Traducir al castellano el texto siguiente:

Systèmes de taille.

Les vignes sont formées selon différents systèmes de taille [...] qui se distinguent par la disposition dans l'espace du tronc, des bras et des bois de taille. Les systèmes de taille les plus courants sont les suivants:

- la souche simple, constituée d'un tronc prolongé par un bras rudimentaire portant un ou deux bois de taille; le plus souvent le bras porte une branche fructifère et un courson de retour [...];

- l'éventail, constitué par un tronc et deux ou plusieurs bras divergents partant d'un même point et disposés dans un même plan, le plus souvent vertical [...] ou parfois oblique [...];

- le cordon, constitué par un tronc dont une partie, disposée horizontalement, porte des coursons [...] ou des longs bois ou une taille mixte;

- le gobelet, constitué d'un tronc et de bras répartis dans l'espace portant une ou plusieurs pousses fructifères généralement à coursons [...], plus rarement à longs bois.

(Reynier, 2000: pp. 305-306). 
